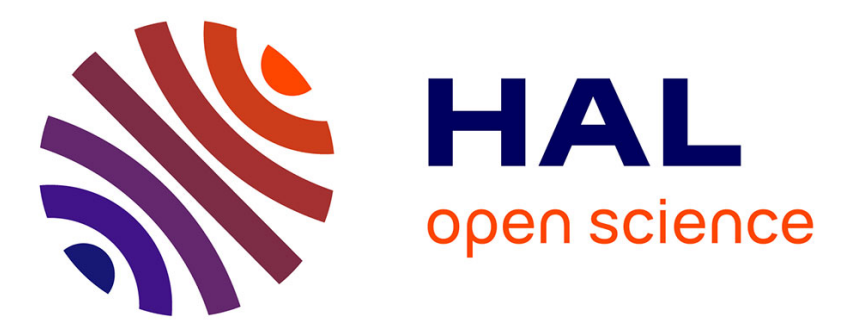

\title{
Arrhythmic dynamics from singularity analysis of electrocardiographic maps
}

Oriol Pont, Hussein Yahia, Binbin Xu

\section{To cite this version:}

Oriol Pont, Hussein Yahia, Binbin Xu. Arrhythmic dynamics from singularity analysis of electrocardiographic maps. EBMC'13-The 35th Annual International Conference of the IEEE Engineering in Medicine and Biology Society, IEEE, Jul 2013, Osaka, Japan. 10.1109/EMBC.2013.6610955 . hal-00823856

\section{HAL Id: hal-00823856 https://hal.inria.fr/hal-00823856}

Submitted on 18 May 2013

HAL is a multi-disciplinary open access archive for the deposit and dissemination of scientific research documents, whether they are published or not. The documents may come from teaching and research institutions in France or abroad, or from public or private research centers.
L'archive ouverte pluridisciplinaire HAL, est destinée au dépôt et à la diffusion de documents scientifiques de niveau recherche, publiés ou non, émanant des établissements d'enseignement et de recherche français ou étrangers, des laboratoires publics ou privés. 


\title{
Arrhythmic dynamics from singularity analysis of electrocardiographic maps
}

\author{
Oriol Pont ${ }^{1,2}$, Hussein Yahia ${ }^{1}$, Binbin $\mathrm{Xu}^{1,2}$ \\ ${ }^{1}$ Team GeoStat - INRIA Bordeaux Sud-Ouest, 33405 Talence, France \\ ${ }^{2}$ L'Institut de rythmologie et modélisation cardiaque LIRYC, U. Bordeaux, 33000 Bordeaux \\ \{oriol.pont, hussein.yahia, binbin.xu\}einria.fr
}

\begin{abstract}
From a point view of nonlinear dynamics, the electrical activity of the heart is a complex dynamical system, whose dynamics reflects the actual state of health of the heart. Nonlinear signal-processing methods are needed in order to accurately characterize these signals and improve understanding of cardiac arrhythmias. Recent developments on reconstructible signals and multiscale information content show that an analysis in terms of singularity exponents provides compact and meaningful descriptors of the structure and dynamics of the system. Such approach gives a compact representation atrial arrhythmic dynamics, which can sharply highlight regime transitions and arrhythmogenic areas.
\end{abstract}

\section{Introduction}

The electrical activity of the human heart is a complex system. The heart rhythm is formed through complex synchronization processes between pacemaker cells and so it shows chaotic rate fluctuations. Compared to the average interbeat intervals, these fluctuations are significantly smaller, so the sinus rhythm appears as mainly periodic, but fluctuations around this main period follow structured nontrivial dynamics. In fact, it has been reported that interbeat fluctuations have a multifractal scale-invariant structure $[1,2,3]$. The resulting signal reflects the network topology generating it, for which the methods of analysis of dynamic structure are especially suitable. In particular, an analysis based on the singularity exponents and the optimal wavelet allows a direct access to the geometric characteristics of the multiscale behavior. This methodology is known to give more accurate estimation of the tails of the singularity spectrum and is generally more robust on empirical data. Having accurate estimates is of paramount importance to anticipate as much as possible when the heartbeat starts to drift from the healthy behavior $[4,5]$.

Altering the electrical activity can restore the heart to a healthy sinus rhythm. For example, in case of paroxysmal AF, Haïssaguerre et al. have shown [6] that for $80 \%$ of patients, electrical insulation of the pulmonary veins allows the patient to regain a normal heart rhythm $[7,8,9,10,11,12,13]$. But in persistent or permanent $\mathrm{AF}$, the location of pathogen areas remains difficult and is still an open problem. So, the characterization of these signals is of vital importance to detect signs of transition to an arrhythmia and to understand the arrhythmia mechanisms, which are at the theoretical foundations of the therapies by electrical insulation.

The paper is structured as follows: the next Section 2 introduces the basics of our singularity analysis method to process the cardiac electrical signals in different dimensions. In Section 3 we present the analysis on atrial fibrillation data and discuss how our methods identify dynamical changes in cardiac rhythm. Finally, in Section 4 we draw the conclusions of our work.

\section{Singularity analysis method}

The method of singularity analysis comes from the Microcanonical Multiscale Formalism (MMF) [14] which is a theoretical and methodological framework for the analysis of multiscale signals. These singularity exponents describe the local regular / singular behavior of the signal around each point, i.e. how rare or unreconstructible is the value at that point from the rest of the signal. A reconstruction kernel that is deterministic, linear, isotropic and translational invariant is uniquely defined and its form implies locally evaluated singularities and thus no need to assume any kind of stationarity $[14,15,16]$. Given a signal $s(\mathbf{x})$, its singularity exponent $h(\mathbf{x})$ can be determined, when the following condition is fulfilled :

$$
\mathscr{T}_{\Psi} \mu(\mathbf{x}, r)=\alpha_{\Psi}(\mathbf{x}) r^{h(\mathbf{x})}+o\left(r^{h(\mathbf{x})}\right) \quad(r \rightarrow 0)
$$


where $\mathscr{T}_{\Psi} \mu(\mathbf{x}, r)=\int_{\mathbb{R}^{d}} \mathrm{~d} \mu\left(\mathbf{x}^{\prime}\right) \Psi\left(\left(\mathbf{x}-\mathbf{x}^{\prime}\right) / r\right)$ is the wavelet projection of the measure $\mu$ at point $\mathbf{x}$ (spatial or temporal) and scale $r, \mathrm{~d} \mu(\mathbf{x})=\|\nabla s\|(\mathbf{x}) \mathrm{d} \mathbf{x}$ is the gradient-modulus measure and $\Psi$ is a certain kernel known as mother wavelet.

The orientation of singularities defines a fast and simple dynamics which, for the case of heartbeat, is statistically compatible with a stochastic process without memory $[17,5]$. The key manifold of the signal driving its dynamics is called the oriented most-singular component. So, reconstructing the signal by a reconstruction kernel [14] only from that oriented manifold results in a reduced signal, which follows the same behavior (1) with the same exponents but different prefactor $\alpha(\mathbf{x})$ and secondary terms. The modulation factor linking the original with the reduced signal defines a complex but slow dynamics called source field (SF):

$$
\mu_{S}(\mathscr{A})=\int_{\mathscr{A}} \mathrm{d} \mu_{r}(\vec{x}, t) \operatorname{SF}(\vec{x}, t)
$$

where $r$ refers to the reduced signal. This allows defining the SF as the Radon-Nikodym derivative between the measure on the signal and that on the reduced signal [4],

$$
S F(\vec{x}, t)=\frac{\mathrm{d} \mu_{s}}{\mathrm{~d} \mu_{r}}(\vec{x}, t) .
$$

\section{Results on ECG, endocavitary catheter measures and electric potential maps}

First we check the validity of the formalism proposed in the previous section and apply it to different types of signal describing the electrical activity of the human heart. Namely, 1D time series of electric potential as measured by electrodes in catheters inside the heart, measured on the body surface (ECG), and $2 \mathrm{D}+t$ maps from non-invasive body surface measurements projected on an epicardial mesh. The formalism adapts to numerical methods for each case.

Data All the processed signals correspond either to healthy sinus rhythm or to cases of atrial arrhythmias, namely atrial flutter and atrial fibrillation. Time series: we have processed electric potential measures (unipolar and bipolar) recorded during radiofrequency ablation operations at Haut-Leveque hospital. They include 4 channels for electrocardiogram measurements on the skin and 17 channels from electrodes in catheters used for the ablation procedure. Recordings include sinus rhythm and atrial fibrillation [18]. We have also processed signals \#201 and \#217 of MIT-BIH Arrhythmia Database [19, 20]. Electrocardiographic maps: Body surface measurements by electrodes all around the thorax. A scan of the heart geometry and relative position to the electrodes is used to project an inverse mapping of the electric potential on the epicardium [21, 22, 23]. Data come from two patients, the one under atrial flutter and the other under atrial fibrillation $[4,5]$.

Potential time series analysis In the time domain, SF varies infrequently and exhibits sharp transitions, Fig. 1. AF presents a distinctive dynamical character from sinus rhythm. Transitions concentrate in points where reconstruction deviates more, meaning that information concentrates on these points and SF transitions correspond to actual changes in the dynamical properties of the signal [5].
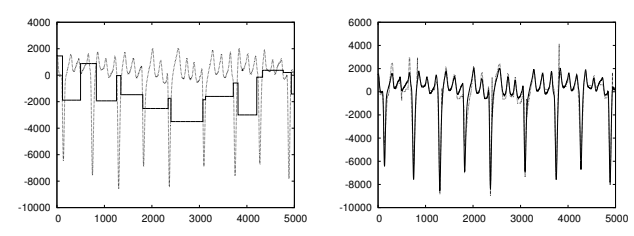

Figure 1. V1 lead under AF. Left: SF (solid) vs. original (dashed). Right: reconstruction (dashed) vs. original (solid). Reconstruction excels, as we capture most of the dynamics.

Source field on potential maps Fig. 2 shows the SF on a complex AF case with multiple problematic areas. Extreme SF values sharply highlight the arrhythmogenic areas whose ablation stopped the fibrillation. Small SF values correspond to less fluctuation modulation and so a more prominent role of the fast dynamics, just the opposite behavior to the case of a simpler flutter reentry [5].

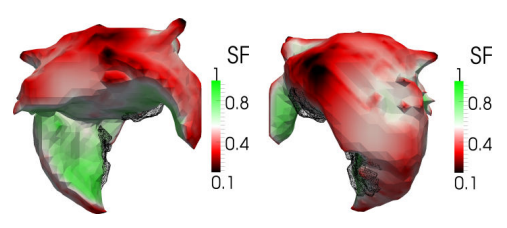

Figure 2. Average SF maps on atria under AF (valves in black), it sharply highlights key fibrillation areas: pulmonary veins and the left posterior inferior area, whose ablation stopped AF.

Dynamical attractor A different approach that nonetheless also provides accurate complexity measures in cardiodynamics is the dynamic phase-space reconstruction implied by the embedding theorem [24, 25]. 
The principle of this approach relate the properties of a time series with the topological properties of an object embedded in a space where all the states of the dynamics are uniquely represented. Since that constructed space is topologically equivalent to the $m \mathrm{D}$ phase space of the dynamics in abstract coordinates, we can construct it with $m$ independent observations. A simple way to achieve this consists of finding a time lag for which observations do not mutually influence, but to discriminate changes of regime we take lags only at the local neighborhood of each point. At each point, the embedding dimension $m$ is the least one that embeds the dynamics (which is twice plus one the Minkowski dimension of its attractor set) and the time lag $\tau$ is the minimum for which the $m$ coordinates are independent. As with singularity analysis, these methods are robust and well adapted to discretized, real-world signals, they render compact descriptions of their dynamics, and they characterize complexity degree and information distribution [26, 27]. Case \#217 of MIT-BIH Arrhythmia Database displays a fragmented electrogram with many intermittent episodes of atrial flutter and atrial fibrillation. Embedding dimensions do not evolve significantly, but time lags reveal a strong correlation of their fluctuations with the arrhythmic episodes, Fig. 3 [28].

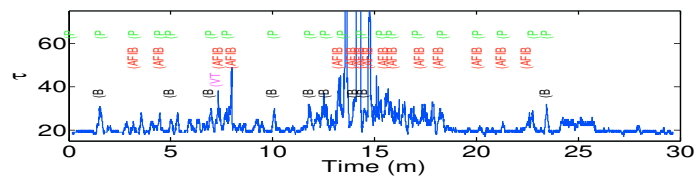

Figure 3. Time lags (in samples, at $360 \mathrm{~Hz}$ ) of phase-space reconstruction for MIT-BIH Arrhythmia Database case \#217.

\section{Discussion and conclusions}

Multiscale nonlinear analysis provides appropriate tools to characterize cardiac dynamics. This is the case both for electric potential time series (body surface and endocavitary) as well as for electrocardiographic images mapped on the surface of the heart. In this paper, we have presented how this type of signal analysis can be exploited to provide meaningful complexity measures with minimal assumptions on the underlying dynamics.

To that extent, independently of the signal type, singularity analysis allows a canonical decoupling of a complexly structured slow dynamics from a transitional fast dynamics that is modulated by the former. The fast dynamics reduces to a three-state Markov process whose eigenvalues indicate atrial arrhythmic regimes. The slow dynamics is stochastically more complex and it describes a Radon-Nikodym derivative of the actual signal from what can be inferred by the fast dynamics alone. The slow dynamics has small fluctuations and abrupt changes that indicate dynamical transitions in time and space, highlighting arrhythmogenic areas.

The robustness of the approach can be corroborated by comparing it with an independent analysis method. In that sense, we have performed phase-space reconstructions of the chaotic signals by means of the embedding theorem. We observe a correspondence of time lag fluctuations of such reconstructions with atrial fibrillation episodes in the same way as with the dynamical changes coming from singularity exponents. These results open the way for improved model-independent complexity descriptors to be used as non-invasive diagnosis support or operation guide in cases of cardiac arrhythmias, particularly atrial fibrillation.

\section{Acknowledgements}

B. $\mathrm{Xu}$ is financially supported by the French IHU in cardiac rhythmology L'Institut de rythmologie et modélisation cardiaque LIRYC and the regional council of Aquitaine. We thank CHU Bordeaux - HautLeveque hospital for providing the endocavitary data and we thank CHU Bordeaux - Haut-Leveque hospital, IHU LIRYC and the CardioInsight Technologies company for the epicardial electrocardiographic maps.

\section{References}

[1] R. I. Kitney and O. Rompelman, The Study of heart-rate variability / edited by R. I. Kitney and O. Rompelman. Clarendon Press ; Oxford University Press, Oxford : New York :, 1980.

[2] M. Kobayashi and T. Musha, "1/f fluctuation of heartbeat period," Biomedical Engineering, IEEE Transactions on, vol. BME-29, pp. 456 -457, june 1982.

[3] C.-K. Peng, J. Mietus, J. M. Hausdorff, S. Havlin, H. E. Stanley, and A. L. Goldberger, "Long-range anticorrelations and non-gaussian behavior of the heartbeat," Phys. Rev. Lett., vol. 70, pp. 1343-1346, Mar 1993.

[4] O. Pont, H. Yahia, R. Dubois, and M. Haissaguerre, "A Singularity-analysis Approach to characterize Epicardial Electric Potential," in Computing in Cardiology, (Krakow, Poland), 2012.

[5] O. Pont, M. Haissaguerre, H. Yahia, N. Derval, and M. Hocini, "Microcanonical processing methodology for ECG and intracardial potential: application to atrial fibrillation," Transactions on Mass-Data Analysis of Images and Signals, vol. 3, no. 1, pp. 15-34, 2011.

[6] M. Haïssaguerre, P. Jaïs, D. C. Shah, A. Takahashi, M. Hocini, G. Quiniou, S. Garrigue, A. Le Mouroux, 
P. Le Métayer, and J. Clémenty, "Spontaneous initiation of atrial fibrillation by ectopic beats originating in the pulmonary veins," New England Journal of Medicine, vol. 339, pp. 659-666, Sept. 1998.

[7] R. Dubois, P. Roussel, M. Hocini, F. Sacher, M. Haissaguerre, and G. Dreyfus, "A wavelet transform for atrial fibrillation cycle length measurements," in Computers in Cardiology, 2009, pp. 501 -504, sept. 2009.

[8] P. Sanders, C. J. Nalliah, R. Dubois, Y. Takahashi, M. Hocini, M. Rotter, T. Rostock, F. Sacher, L.-f. Hsu, A. Jönsson, M. D. O'neill, P. Jaïs, and M. Haïssaguerre, "Frequency mapping of the pulmonary veins in paroxysmal versus permanent atrial fibrillation," Journal of Cardiovascular Electrophysiology, vol. 17, no. 9, pp. 965972, 2006.

[9] Y. Takahashi, P. Sanders, P. Jaïs, M. Hocini, R. Dubois, M. Rotter, T. Rostock, C. J. Nalliah, F. Sacher, J. Clémenty, and M. Haïssaguerre, "Organization of frequency spectra of atrial fibrillation: Relevance to radiofrequency catheter ablation," Journal of Cardiovascular Electrophysiology, vol. 17, no. 4, pp. 382-388, 2006.

[10] M. Hocini, P. Jais, P. Sanders, Y. Takahashi, M. Rotter, T. Rostock, L.-F. Hsu, F. Sacher, S. Reuter, J. Clementy, and M. Haissaguerre, "Techniques, evaluation, and consequences of linear block at the left atrial roof in paroxysmal atrial fibrillation: a prospective randomized study.," Circulation, vol. 112, pp. 3688-3696, Dec 2005.

[11] M. Hocini, P. Sanders, P. Jaïs, L.-F. Hsu, R. Weerasoriya, C. Scavée, Y. Takahashi, M. Rotter, F. Raybaud, L. Macle, J. Clémenty, and M. Haïssaguerre, "Prevalence of pulmonary vein disconnection after anatomical ablation for atrial fibrillation: consequences of wide atrial encircling of the pulmonary veins," European Heart Journal, vol. 26, no. 7, pp. 696-704, 2005.

[12] M. Hocini, P. Jais, F. Sacher, S. Reuter, J. Clementy, and M. Haissaguerre, "Mapping and ablation of malignant ventricular arrhythmias," Arch Mal Coeur Vaiss, vol. 98, pp. 34-41, Dec 2005.

[13] M. Haissaguerre, F. Extramiana, M. Hocini, B. Cauchemez, P. Jais, J. A. Cabrera, G. Farre, A. Leenhardt, P. Sanders, C. Scavee, L.-F. Hsu, R. Weerasooriya, D. C. Shah, R. Frank, P. Maury, M. Delay, S. Garrigue, and J. Clementy, "Mapping and ablation of ventricular fibrillation associated with long-qt and brugada syndromes," Circulation, vol. 108, no. 8, pp. 925-928, 2003.

[14] A. Turiel, H. Yahia, and C. Pérez-Vicente, "Microcanonical multifractal formalism: a geometrical approach to multifractal systems. Part I: Singularity analysis," Journal of Physics A, vol. 41, p. 015501, 2008.

[15] O. Pont, A. Turiel, and C. Perez-Vicente, "On optimal wavelet bases for the realization of microcanonical cascade processes," Int. J. Wavelets Multi., IJWMIP, vol. 9, pp. 35-61, January 2011.

[16] O. Pont, A. Turiel, and H. Yahia, "An optimized algorithm for the evaluation of local singularity exponents in digital signals," in 14th International Workshop, IWCIA 2011 (J. Aggarwal, R. Barneva, V. Brimkov, K. Ko- routchev, and E. Korutcheva, eds.), vol. 6636 of Lecture Notes in Computer Science (LNCS), (Madrid, Spain), pp. 346-357, Springer, 2011.

[17] O. Pont, M. Haissaguerre, H. Yahia, N. Derval, and M. Hocini, "Heartbeat dynamics from a microcanonical multifractal approach," in Computing in Cardiology, vol. 38, 2011.

[18] O. Pont, M. Haissaguerre, H. Yahia, N. Derval, and M. Hocini, "A novel analysis method to characterize heartbeat dynamics through the microcanonical multiscale formalism," in Advances in Mass Data Analysis of Images and Signals in Medicine, Biotechnology, Chemistry and Food Industry (P. Perner, ed.), (Fockendorf, Germany), pp. 50-62, ibai Publishing, 2011.

[19] G. B. Moody and R. G. Mark, "The impact of the mit-bih arrhythmia database.," IEEE Eng Med Biol Mag, vol. 20, no. 3, pp. 45-50, 2001.

[20] A. L. Goldberger, L. A. N. Amaral, L. Glass, J. M. Hausdorff, P. C. Ivanov, R. G. Mark, J. E. Mietus, G. B. Moody, C.-K. Peng, and H. E. Stanley, "Physiobank, physiotoolkit, and physionet : Components of a new research resource for complex physiologic signals," Circulation, vol. 101, no. 23, pp. e215-e220, 2000.

[21] C. Ramanathan, R. N. Ghanem, P. Jia, K. Ryu, and Y. Rudy, "Noninvasive electrocardiographic imaging for cardiac electrophysiology and arrhythmia," Nat Med, vol. 10, pp. 422-428, Apr. 2004.

[22] P. Jia, C. Ramanathan, R. N. Ghanem, K. Ryu, N. Varma, and Y. Rudy, "Electrocardiographic imaging of cardiac resynchronization therapy in heart failure: Observation of variable electrophysiologic responses," Heart Rhythm, vol. 3, pp. 296-310, Mar. 2006.

[23] P. S. Cuculich, Y. Wang, B. D. Lindsay, M. N. Faddis, R. B. Schuessler, R. J. Damiano, L. Li, and Y. Rudy, "Noninvasive characterization of epicardial activation in humans with diverse atrial fibrillation patterns / clinical perspective," Circulation, vol. 122, no. 14, pp. 13641372, 2010.

[24] O. Fojt and J. Holcik, "Applying nonlinear dynamics to ecg signal processing," Engineering in Medicine and Biology Magazine, IEEE, vol. 17, pp. 96 -101, march-april 1998.

[25] R. B. Govindan, K. Narayanan, and M. S. Gopinathan, "On the evidence of deterministic chaos in ecg: Surrogate and predictability analysis.," Chaos, vol. 8, pp. 495-502, Jun 1998.

[26] B. Xu, S. Jacquir, G. Laurent, J.-M. Bilbault, and S. Binczak, "A hybrid stimulation strategy for suppression of spiral waves in cardiac tissue," Chaos, Solitons and Fractals, vol. 44, pp. 633-639, Aug. 2011.

[27] S. Jacquir, S. Binczak, B. Xu, G. Laurent, D. Vandroux, P. Athias, and J.-M. Bilbault, "Investigation of micro spiral waves at cellular level using a microelectrode arrays technology," International Journal of Bifurcation and Chaos, vol. 21, pp. 209-223, Jan. 2011.

[28] B. Xu and O. Pont, "Chaotic cardiodynamics phase-space reconstruction as a detector for attrial arrhythmias.” In preparation for Nonlinear Analysis, 2013. 